\title{
Polarization of $\mathrm{H}_{2} \mathrm{O}$ masers in the presence of velocity and magnetic field gradients
}

\begin{abstract}
W. H. T. Vlemmings ${ }^{1}$
Jodrell Bank Observatory, University of Manchester, Macclesfield, Cheshire, SK11 9DL, UK

e-mail: wouter@jb.man.ac.uk

Received 21 July 2005 / Accepted 12 September 2005

\section{ABSTRACT}

Through polarization observations $\mathrm{H}_{2} \mathrm{O}$ masers are excellent probes of magnetic fields in the maser region. Magnetic field strengths, such as those in the $\mathrm{H}_{2} \mathrm{O}$ masers regions of the envelopes of late-type stars and star-forming regions, are typically determined using a direct relation between the field strength and the observed circular polarization. Here it is shown that velocity and magnetic field gradients along the maser have a significant effect on the field strengths obtained from circular polarization observations. Due to velocity gradients the actual magnetic field strength could be up to $100 \%$ higher than the field strength derived from the observations. Additionally, when a magnetic field gradient is present, the resulting circular polarization derived is caused predominantly by the average magnetic field in the unsaturated maser core. Measurements of the fractional linear polarization are not affected by velocity or magnetic field strength gradients, though changes in the magnetic field angle along the maser do quench the linear polarization intensity when the maser saturates.
\end{abstract}

Key words. masers - radiative transfer - polarization - magnetic fields

\section{Introduction}

Astrophysical $\mathrm{H}_{2} \mathrm{O}$ masers, like other maser species such as $\mathrm{OH}$ and $\mathrm{SiO}$, are excellent probes of magnetic fields in a variety of interesting regions. The magnetic field strength is derived from observations of maser polarization caused by Zeeman splitting. Polarization observations of the $22 \mathrm{GHz} \mathrm{H_{2 }} \mathrm{O}$ masers in the circumstellar envelopes (CSEs) around evolved stars have made important contributions to the understanding of the magnetic fields of evolved stars (Vlemmings et al. 2002, 2005, hereafter $\mathrm{V02}$ and V05a). $\mathrm{H}_{2} \mathrm{O}$ maser observations have also provided information on the strength and structure of the magnetic field in star-forming regions (Fiebig \& Guesten 1989; Sarma et al. 2001, 2002) and have provided upper limits for the field in the megamaser galaxy NGC 4258 (Modjaz et al. 2005).

However, the exact relationship between the observed maser polarization and the magnetic field strength is not straightforward, leading to uncertainties in the determined field strength. It was shown in Nedoluha \& Watson (1992, hereafter NW92) that the influence of the different hyperfine components of the $\mathrm{H}_{2} \mathrm{O}\left(6_{16}-5_{23}\right)$ rotational transition as well as of the degree of maser saturation is significant. The effect of maser saturation on the magnetic field strength determination can be as large as a factor of 2 (NW92). Additionally, velocity and magnetic field gradients might play an important role. While typical $\mathrm{H}_{2} \mathrm{O}$ maser spot sizes are between $\sim 10^{12}$ and $10^{13} \mathrm{~cm}$
(Reid \& Moran 1981; Imai et al. 1997), the actual size of the maser region will be several times larger due to beaming (e.g. Goldreich \& Keeley 1972). As a result the maser path length could be as long as $10^{14}-10^{15} \mathrm{~cm}$. Across such a path length, velocity and magnetic field gradients likely influence the observed polarization. In Vlemmings \& van Langevelde (2005, hereafter V05b) it was shown that velocity gradients alter the shape of the total intensity line profile. From this analysis and from earlier observations (e.g. Richards et al. 1999) it is apparent that in CSEs velocity shifts of $\sim 1 \mathrm{~km} \mathrm{~s}^{-1}$ are common. In this paper the effects of velocity and magnetic field gradients along the maser path on the magnetic field strength determined from circular polarization measurements and on the observed fractional linear polarization are examined.

\section{Background}

Similar to the analysis in V02 and V05b, the equations of state for the populations of the upper $\left(6_{16}\right)$ and lower $\left(5_{23}\right)$ rotational levels of the three strongest hyperfine components $\left(F-F^{\prime}=\right.$ $7-6,6-5$ and 5-4) of the $22 \mathrm{GHz} \mathrm{H}_{2} \mathrm{O}$ maser have been solved using the method described in NW92. The equation of state for the number density $n(F, a, v)$ of the upper energy levels $(F)$ is

$$
\begin{aligned}
0= & \lambda_{F}\left(v_{\mathrm{s}}\right)-\left(\Gamma+\Gamma_{v}\right) n(F, a, v) \\
& +R\left(F, F^{\prime}, a, b, v\right)\left(n\left(F^{\prime}, b, v\right)-n(F, a, v)\right) \\
& +\phi\left(v_{\mathrm{s}}\right)\left(\frac{\Gamma_{v}}{\sum g_{F}}\right) \int \mathrm{d} v \sum g_{F} n(F, a, v) .
\end{aligned}
$$


The equation for the number density $n\left(F^{\prime}, b, v\right)$ of the lower levels $\left(F^{\prime}\right)$ is similar but reversed. Here $a$ and $b$ denote the different magnetic sub-states of each hyperfine component. The population levels are solved as a function of molecular velocity $v$ and at different positions along the maser propagation direction. The statistical weights are designated by $g_{F}$ and $g_{F}^{\prime}$, and are from Kukulich (1969). The pump rate $\lambda_{F}\left(v_{\mathrm{s}}\right)$ is assumed to be the same for the different hyperfine components and has a Maxwellian distribution. The results of the calculations depend only on the the ratio $\left(\lambda_{F}-\lambda_{F}^{\prime}\right) / \lambda_{F}$, which is of the order of a few percent (Anderson \& Watson 1993). Generally $v_{\mathrm{s}}=v$, but when velocity gradients are introduced later, the molecular velocity of the maser pump $v_{\mathrm{s}}$ changes along the maser path. The rate for stimulated emission $R\left(F, F^{\prime}, a, b, v\right)$ (hereafter $R$ ) is calculated using the local maser intensity and the hyperfine interaction coefficients as described in NW92. $\Gamma$ is the decay rate for the molecular excitations. The crossrelaxation rate $\Gamma_{v}$ describes the reabsorbtion of previously emitted infrared pump photons trapped in optically thick transitions of the maser system, which generate a newly excited molecule at random, within a Maxwellian velocity distribution $\left(\phi\left(v_{\mathrm{s}}\right)\right)$. Elastic collisions between the maser molecules and intermixed $\mathrm{H}_{2}$ molecules have not been included. The effects of these are described in detail in Elitzur (1990) and are unimportant for the $\mathrm{H}_{2} \mathrm{O}$ masers discussed here.

The maser intensity, which is solved using the radiative transfer equations from NW92, influences the level populations through $R$. However, as it is itself dependent on the level populations determined with Eq. (1), the maser total intensity $(I)$, linear polarization $(Q)$ and circular polarization $(V)$ are solved iteratively along the maser path. Assuming nearly one-dimensional maser propagation the beaming of the maser radiation is represented by the solid angle $\Delta \Omega$. The emerging maser fluxes will thus be represented in $T_{\mathrm{b}} \Delta \Omega$, with $T_{\mathrm{b}}$ the brightness temperature. All calculations were performed with a brightness temperature for the radiation incident on the maser region of $T_{\mathrm{b}} \Delta \Omega=0.1 \mathrm{~K}$ sr. It was verified in NW92 and V02 that the results are insensitive to the chosen initial value. The calculations are performed for different thermal line widths $\left(v_{\text {th }}\right)$ of the Maxwellian particle velocity distribution, where $v_{\text {th }} \approx 0.5(T / 100)^{1 / 2} \mathrm{~km} \mathrm{~s}^{-1}$ with $T$ the temperature of the masing molecules in K. Nedoluha \& Watson (1991) and NW92 have shown that when Eq. (1) is solved without including the contribution of the cross-relaxation rate $\Gamma_{v}$, the results for the line profiles, fractional polarizations and line width in the case of non-negligable $\Gamma_{v}$ can be obtained by scaling the results with $\left[\Gamma+\Gamma_{v}\right]$. Following this result, the calculations in this paper are performed for $\Gamma=1 \mathrm{~s}^{-1}$ and $\Gamma_{v}=0 \mathrm{~s}^{-1}$. Thus, in all the results of this paper, the emerging brightness temperature $T_{\mathrm{b}} \Delta \Omega$ can be replaced with $T_{\mathrm{b}} \Delta \Omega \times\left[\Gamma+\Gamma_{v}\right]$ to obtain the results for different values of the decay and cross-relaxation rates.

To solve the equations of state it is assumed that the Zeeman frequency shift $g \Omega$ is much greater than $R, \Gamma$ and $\Gamma_{v}$. Then the off-diagonal elements of the density matrix describing the molecular states are negligible, greatly simplifying the calculations. For a magnetic field strength of $\sim 1.0 \mathrm{G}$, $g \Omega$ of the dominant hyperfine transitions is $\sim 10^{4} \mathrm{~s}^{-1}$ while for $T_{\mathrm{b}} \Delta \Omega<10^{12} \mathrm{~K}$ sr the rate for stimulated emission $R \leq$ $100 \mathrm{~s}^{-1}$ (V02). Most of the calculations in this paper are performed for a magnetic field strength of $B=1 \mathrm{G}$. For the highest $T_{\mathrm{b}} \Delta \Omega$, the results will be valid for fields down to $\sim 10 \mathrm{mG}$, when $g \Omega$ becomes comparable to $R$. However, the low observed linear polarization of the $22 \mathrm{GHz} \mathrm{H}_{2} \mathrm{O}$ masers (e.g. V02, V05a) indicates that $T_{\mathrm{b}} \Delta \Omega$ is at most $10^{11} \mathrm{~K}$ sr and typically even lower. Thus, the results presented in this paper are valid for $B$ well below $1 \mathrm{mG}$.

$22 \mathrm{GHz} \mathrm{H}_{2} \mathrm{O}$ masers begin to saturate when $R / \Gamma \gtrsim 1 \mathrm{~s}^{-1}$ and approach full saturation when $R / \Gamma \gtrsim 100 \mathrm{~s}^{-1}$. Thus, for our models with $\Gamma=1 \mathrm{~s}^{-1}$, saturation starts at $T_{\mathrm{b}} \Delta \Omega \gtrsim 10^{10} \mathrm{~K} \mathrm{sr}$ and full saturation occurs for $T_{\mathrm{b}} \Delta \Omega \gtrsim 10^{12} \mathrm{~K}$ sr. The maser spectral line profile (through rebroadening) and the polarization properties of the maser changes at $R=\left(\Gamma+\Gamma_{v}\right)$ (e.g. NW92). While typically for the $22 \mathrm{GHz} \mathrm{H}_{2} \mathrm{O}$ masers this is close to when saturation occurs, a large cross-relaxation rate means the changes will only occur when the maser has already become partly saturated. As $\Gamma_{v}$ for the astrophysical $\mathrm{H}_{2} \mathrm{O}$ masers is at most $\approx 5 \mathrm{~s}^{-1}$ for $\mathrm{H}_{2} \mathrm{O}$ masers at $T \sim 1000 \mathrm{~K}$ and is less than that lower temperatures (Anderson \& Watson 1993), $\Gamma_{v} \approx \Gamma$ is assumed in this paper when discussing unsaturated and saturated masers. It is worth noting that $\mathrm{H}_{2} \mathrm{O}$ maser observations indicate that in actuality $\Gamma$ is between one and two orders of magnitude less than $\Gamma_{v}$ (Anderson \& Watson 1993). However, as our results scale linearly with $\left(\Gamma+\Gamma_{\nu}\right)$ this does not affect the conclusions in this paper.

\section{Radiative transfer modeling results}

\subsection{Circular polarization}

As shown in NW92 and V02, the $V$-spectra produced from the radiative transfer equations are not anti-symmetric. They are also not always directly proportional to the derivative of the $I$-spectrum due to hyperfine interaction as is often assumed. However, as was discussed in V02, it is impossible to directly observe the intrinsic shape of the $V$-spectrum due to instrumental effects and necessary data calibration steps. Still, the percentage of circular polarization $P_{V}$ can be related to the magnetic field strength using

$$
\begin{aligned}
P_{V} & =\left(V_{\max }-V_{\min }\right) / I_{\max } \\
& =2 \cdot A_{F-F^{\prime}} \cdot B_{[\text {Gauss }]} \cos \theta / \Delta \mathrm{v}_{\mathrm{L}}\left[\mathrm{km} \mathrm{s}^{-1}\right] .
\end{aligned}
$$

Here $V_{\max }$ and $V_{\min }$ are the minimum and maximum of the $V$-spectrum. $I_{\max }$ and $\Delta v_{\mathrm{L}}$ are the peak flux and the full width half-maximum ( $F W H M)$ of the $I$-spectrum respectively. $B$ is the magnetic field strength at an angle $\theta$ to the maser propagation direction. While in most analyses of maser circular polarization, the coefficient $A_{F-F^{\prime}}$ is taken to be a fixed value (e.g Fiebig \& Guesten 1989), it was shown in NW92 to depend on the level of maser saturation and the intrinsic thermal velocity $v_{\text {th }}$ of the maser. Figure 6 of V02 shows $A_{F-F^{\prime}}$ for different values of $v_{\text {th }}$ as a function of emerging maser brightness temperature $T_{\mathrm{b}} \Delta \Omega$. For maser brightness temperatures $T_{\mathrm{b}} \Delta \Omega>10^{9} \mathrm{~K}$ sr it was shown in NW92 that the $\cos \theta$ dependence of Eq. (2) breaks down, effectively also introducing a dependence on $\theta$ to $A_{F-F^{\prime}}$. This was later shown in more 


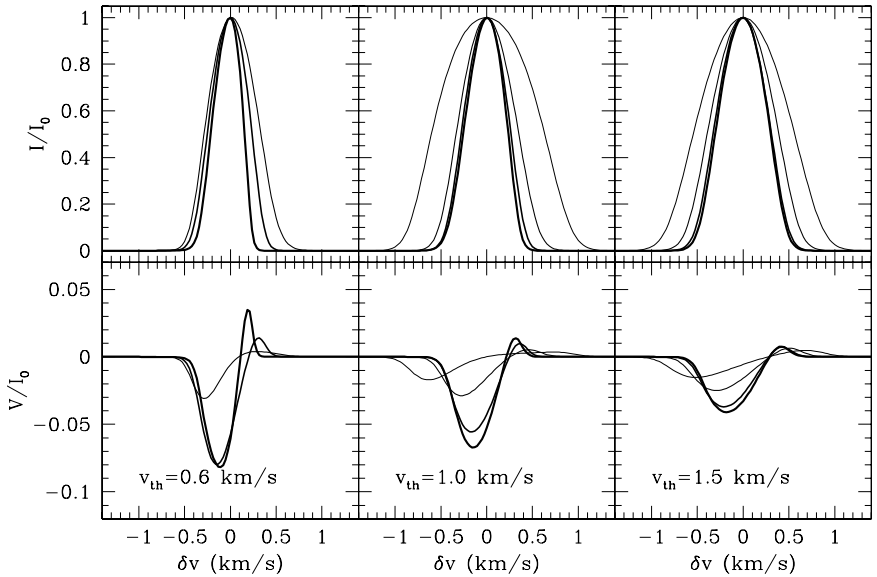

Fig. 1. Normalized total intensity $(I)$ and circular polarization $(V)$ spectra for $v_{\text {th }}=0.6,1.0$ and $1.5 \mathrm{~km} \mathrm{~s}^{-1}$ in the presence of a velocity gradient. From thick to thin the solid lines denote velocity shifts of $\Delta V_{\mathrm{m}}=0,0.5,1.0$ and $1.5 \mathrm{~km} \mathrm{~s}^{-1}$ respectively except for $v_{\text {th }}=0.6 \mathrm{~km} \mathrm{~s}^{-1}$ where $\Delta V_{\mathrm{m}}=1.5 \mathrm{~km} \mathrm{~s}^{-1}$ is omitted.

detail in Watson \& Wyld (2001) for masing involving angular momentum $J=1-0$ and $J=2-1$ transitions. In V02, Fig. 7 shows the derived magnetic field strength dependence on $\theta$ to $A_{F-F^{\prime}}$ for the $22 \mathrm{GHz} J=6-5$ transition.

\subsubsection{Velocity gradients}

First, a velocity gradient is introduced by uniformly shifting, in Eq. (1), the velocity $v_{\mathrm{s}}$ of the pump rate $\lambda_{F}$ for each integration step along the maser path. By varying the amount of shift, a velocity difference $\Delta V_{\mathrm{m}}$ is created between the start and end of the maser amplification path of up to $\sim 1.5 \mathrm{~km} \mathrm{~s}^{-1}$. This corresponds to a velocity gradient of $\Delta V_{\mathrm{m}} / S \mathrm{~km} \mathrm{~s}^{-1} \mathrm{~m}^{-1}$, where $S$ is the length of the maser path. As $S$ depends on the exact values of the pumping rate $\lambda_{F}$, while our results, expressed in emerging brightness temperature are only dependent on the ratio of pumping rates as addressed above, any mention of the velocity gradient will henceforth be referring to the value of $\Delta V_{\mathrm{m}}$. In Nedoluha \& Watson (1988) it was shown that the maser splits into several distinctly separate narrow features when $\Delta V_{\mathrm{m}}$ becomes a few times $v_{\text {th }}$, each of the features becoming an independent maser. The calculations in this paper are therefore limited to values of $\Delta V_{\mathrm{m}}$ up to $\sim 1.5 \mathrm{~km} \mathrm{~s}^{-1}$.

Figure 1 shows the effect of a velocity gradient on the total intensity and circular polarization profiles for three different intrinsic thermal velocities $v_{\text {th }}$, produced for the same magnetic field strength $(1 \mathrm{G})$. While for low $\Delta V_{\mathrm{m}}$, the effect of the velocity gradient on the shape of the $I$-profile is small, the effect on the $V$-spectrum can still clearly be seen. To examine how a velocity gradient influences the magnetic field determination from the circular polarization measurements, the normalized $A_{F-F^{\prime}}$ coefficient from Eq. (2) is plotted in Fig. 2 as a function of velocity gradient for $v_{\text {th }}=0.6$ and $1.5 \mathrm{~km} \mathrm{~s}^{-1}$ and emerging brightness temperatures $T_{\mathrm{b}} \Delta \Omega=10^{9}, 10^{10}$ and $10^{11} \mathrm{~K}$ sr. Because of the normalization, the results are independent of the magnetic field angle $\theta$. For $v_{\text {th }}=0.6 \mathrm{~km} \mathrm{~s}^{-1}$ the effect of the

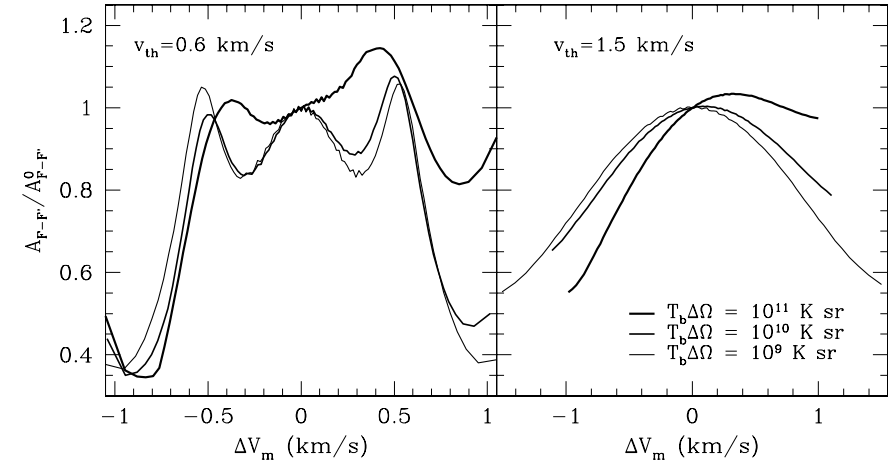

Fig. 2. The dependence of the magnetic field strength determined from circular polarization observation, represented by the $A_{F-F^{\prime}}$ coefficient from Eq. (2), on the magnitude of the velocity gradient $\Delta V_{\mathrm{m}}$ for two values of intrinsic thermal line width $v_{\text {th }} . A_{F-F^{\prime}}$ is normalized by $A_{F-F^{\prime}}^{0}$, which is $A_{F-F^{\prime}}$ for $\Delta V_{\mathrm{m}}=0 \mathrm{~km} \mathrm{~s}^{-1}$. The solid lines represents masers with different emerging brightness temperatures. Note that the noise $(<1 \%)$ on the curves is due to the velocity resolution of our model spectra. The results are independent of the magnetic field angle $\theta$.

hyperfine components is clear. For $T_{\mathrm{b}} \Delta \Omega=10^{9} \mathrm{~K}$ sr none of the hyperfine components are becoming saturated yet, thus $A_{F-F^{\prime}}$ is mostly symmetric around $\Delta V_{\mathrm{m}}=0 \mathrm{~km} \mathrm{~s}^{-1}$. The symmetry breaks down when the hyperfine lines are slowly starting to saturate for $T_{\mathrm{b}} \Delta \Omega \gtrsim 10^{10} \mathrm{~K} \mathrm{sr}$, as maser growth is somewhat inhibited for velocity gradients in the direction of the weaker hyperfine components $\left(F-F^{\prime}=6-5\right.$ and 5-4), while a gradient in the opposite direction allows for stronger maser amplification. As noted above this effect can be postponed further into the saturated regime when $\Gamma_{v}$ is increased. It can be seen in the figure, that $A_{F-F^{\prime}}$ typically decreases for increasing $\left|\Delta V_{\mathrm{m}}\right|$, implying that in the presence of velocity gradients the magnetic field strength $B^{\prime}$ derived with Eq. (2) underestimates the true $B$. For small $v_{\text {th }}$ the field strength can sometimes be underestimated by more than a factor of 2 . However, for a narrow range in $\Delta V_{\mathrm{m}}, A_{F-F^{\prime}}$ is actually enhanced. In those cases $B$ could actually be overestimated by up to $\sim 20 \%$. This effect is largest for the more saturated masers.

\subsection{2. magnetic field gradients}

There are different ways a magnetic field gradient along the maser amplification path can occur. The actual field strength $B$ can change or the angle $\theta$ between the field and the maser propagation direction can vary. Similar to $\Delta V_{\mathrm{m}}$, differences $\Delta \theta$ and $\Delta B$ are introduced between the start and end of the maser path and their influence on the maser polarization is examined. Figure 3 a shows the ratio between the magnetic field $B^{\prime}$ determined with Eq. (2) and the average magnetic field $\langle B\rangle$ along the maser as a function of $T_{\mathrm{b}} \Delta \Omega$ when a gradient $\Delta B$ is included. The shape of this function does not depend on the actual value of $\Delta B$ or on the intrinsic thermal line width $v_{\text {th }}$. While the maser is unsaturated, or more precisely while $R<\Gamma_{v}, B^{\prime}$ is equal to $\langle B\rangle$. However, when the maser saturates, $B^{\prime}$ will be dominated by the average magnetic field in the unsaturated maser region. 

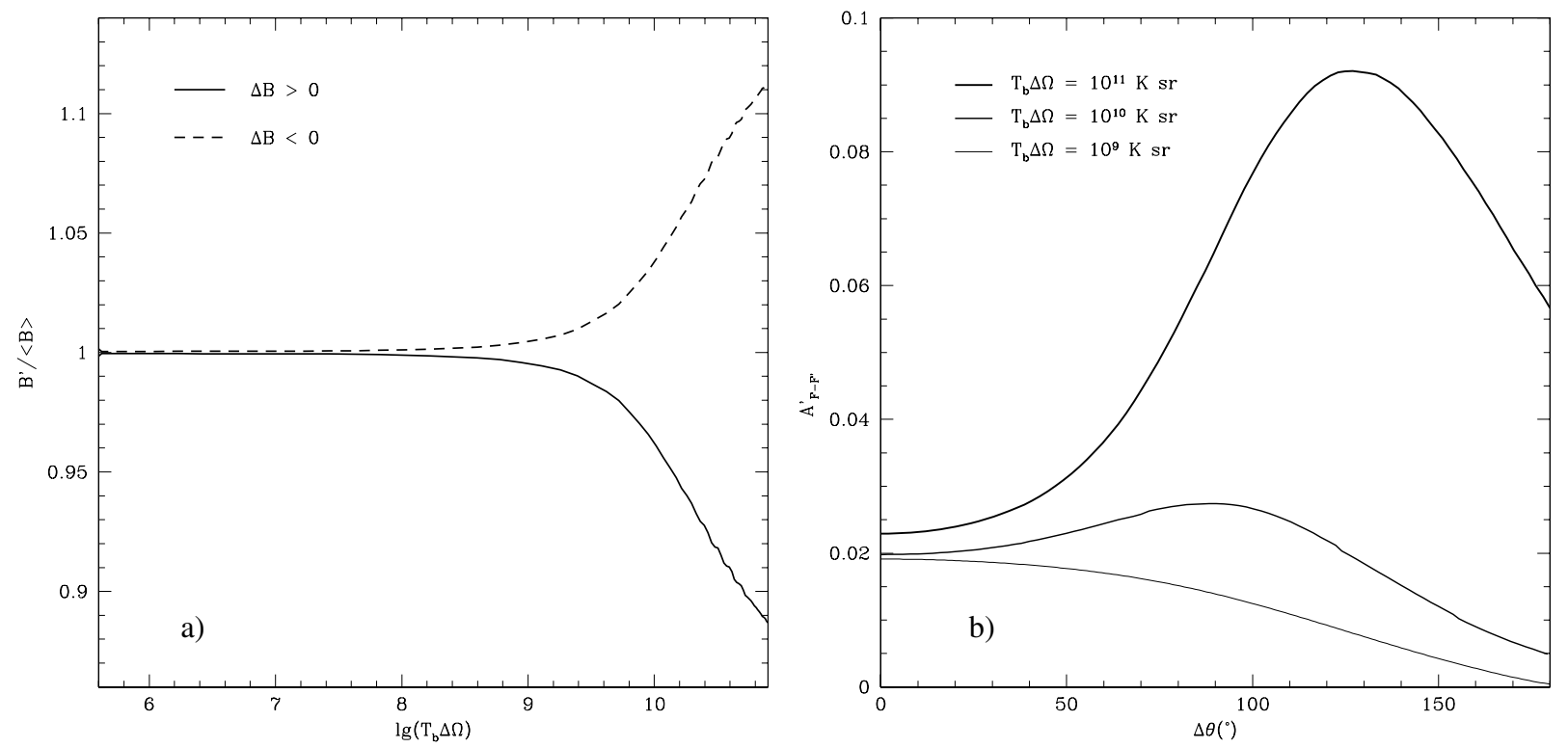

Fig. 3. a) Derived magnetic field strength $B^{\prime}$ over the average field strength along the maser $\langle B\rangle$ as a function of $T_{\mathrm{b}} \Delta \Omega$. The solid and dashed lines correspond to positive and negative magnetic field gradients respectively. b) $A_{F-F^{\prime}}^{\prime}$ as a function of $\Delta \theta$ for different values of emerging brightness temperature $T_{\mathrm{b}} \Delta \Omega$. Calculations were performed for $v_{\mathrm{th}}=0.8 \mathrm{~km} \mathrm{~s}^{-1}$.
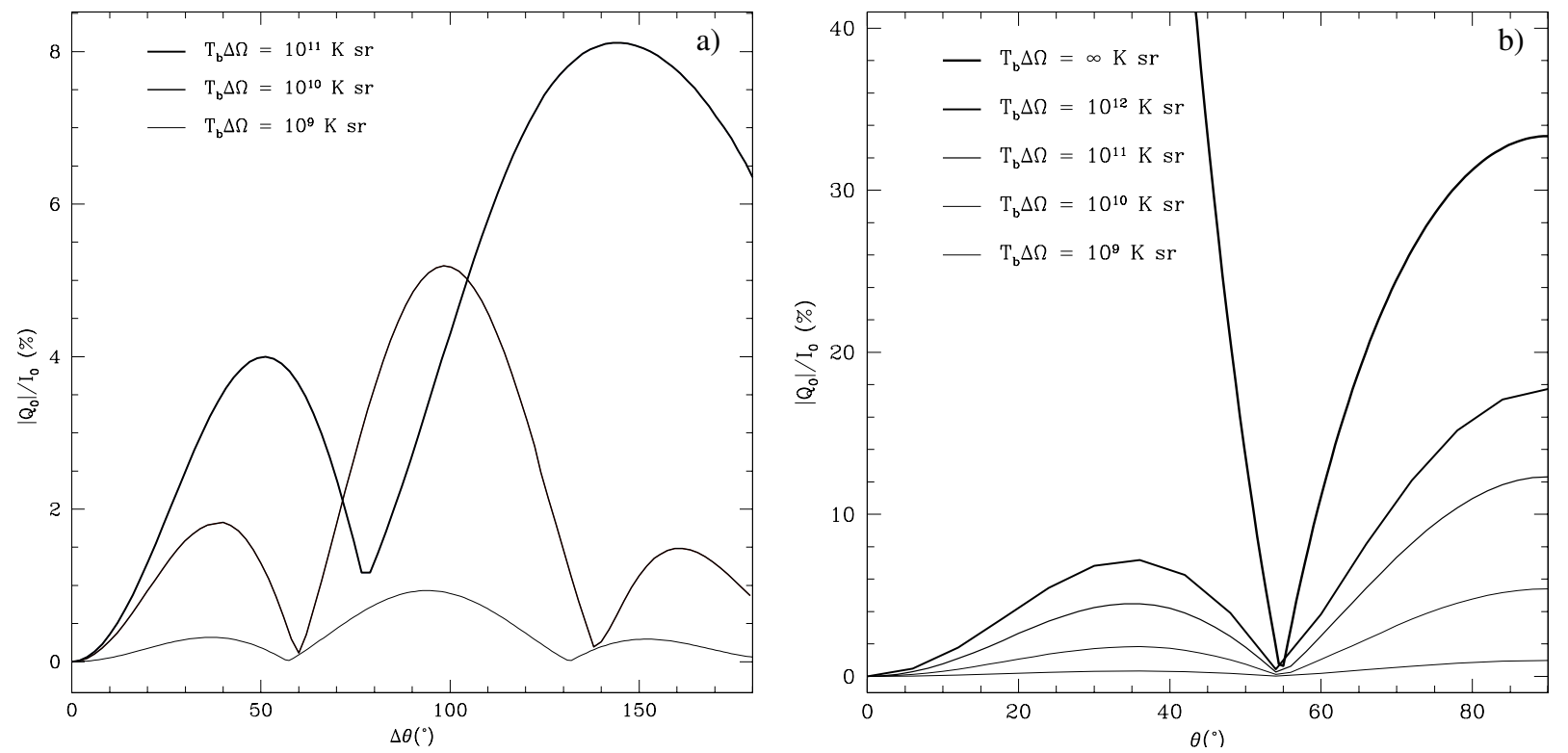

Fig. 4. a) Magnetic field angle gradient $\Delta \theta$ vs. the fractional linear polarization for three different stages of maser saturation and $\theta_{0}=0^{\circ}$. b) The angle $\theta$ between the maser propagation direction and the magnetic field vs. the fractional linear polarization for different values of emerging maser brightness. The thick solid line denotes the theoretical limit from Goldreich et al. (1973) for a completely saturated maser.

Figure $3 \mathrm{~b}$ shows the change in $A_{F-F^{\prime}}^{\prime}$ versus $\Delta \theta$ for different $T_{\mathrm{b}} \Delta \Omega$. The angle $\theta_{0}$, the value of $\theta$ at the start of maser amplification is taken to be $0^{\circ}$. Here $A_{F-F^{\prime}}^{\prime}=A_{F-F^{\prime}} \cos \theta$, as the actual angle $\theta$ cannot be specified due to the inclusion of the gradient $\Delta \theta$. Comparing the unsaturated maser $\left(T_{\mathrm{b}} \Delta \Omega=\right.$ $\left.10^{9} \mathrm{~K} \mathrm{sr}\right)$ with the partly saturated maser $\left(T_{\mathrm{b}} \Delta \Omega=10^{11} \mathrm{~K} \mathrm{sr}\right)$ it is again apparent that, as seen in Fig. 3a, the unsaturated maser regime provides the dominant contribution to the magnetic field strength determined with Eq. (2). Comparing Fig. 3b with Fig. 7 from V02 indicates that the angle average $\langle\theta\rangle$ in the unsaturated maser region determines the observed circular polarization.

\subsection{Linear polarization}

The linear polarization in the presence of a magnetic field was also discussed in NW92. The fractional linear polarization is not found to be affected when including a velocity gradient $\Delta V_{\mathrm{m}}$ or magnetic field gradient $\Delta B$ in the calculations. Only when including a gradient $\Delta \theta$ does the linear polarization change. In Fig. 4a the fractional linear polarization $\left|Q_{0}\right| / I_{0}$ is shown as a function of $\Delta \theta$. This can be compared with $\left|Q_{0}\right| / I_{0}$ as a function of the angle between the magnetic field and the maser propagation direction $\theta$ shown in Fig. $4 \mathrm{~b}$. This relationship, in the limiting case of a completely saturated maser, was 
solved in Goldreich et al. (1973). In the case of an unsaturated maser $\left(T_{\mathrm{b}} \Delta \Omega \lesssim 10^{10} \mathrm{~K} \mathrm{sr}\right)$, the relationship between $\left|Q_{0}\right| / I_{0}$ and the magnetic field angle gradient $\Delta \theta$ is very similar to the relationship between $\left|Q_{0}\right| / I_{0}$ and $\theta$, while when the saturation level increases the linear polarization is quenched and shifted. This indicates that the observed fractional linear polarization is determined by $\theta$ at the end of the unsaturated maser regime, where the largest amplification occurs, and not by the average $\langle\theta\rangle$ over the unsaturated maser core.

\section{Conclusions}

Using a maser radiative transfer code which includes the three strongest hyperfine components of the $22 \mathrm{GHz} \mathrm{H_{2 }} \mathrm{O}_{616}-5_{23}$ rotational transition and their magnetic sub-states, the effects of velocity and magnetic field gradients on the determination of magnetic fields from maser polarization observations have been calculated. It was shown that, due to velocity gradients, the magnetic field strength determined on $\mathrm{H}_{2} \mathrm{O}$ masers in CSEs (V02, V05a), where $v_{\text {th }} \approx 0.8-1.0 \mathrm{~km} \mathrm{~s}^{-1}$ and where velocity gradients of $\sim 1.0 \mathrm{~km} \mathrm{~s}^{-1}$ have been observed (V05b), can be underestimated by up to a factor of 2 . However, for maser features with smaller velocity gradients there will be cases where $B$ is actually overestimated by $\sim 10-20 \%$. The magnetic fields determined on the $\mathrm{H}_{2} \mathrm{O}$ masers in star-formation regions (Fiebig \& Guesten 1989; Sarma et al. 2001, 2002), which have higher thermal line widths $\left(v_{\text {th }} \approx 1.5 \mathrm{~km} \mathrm{~s}^{-1}\right)$ can be underestimated by $\sim 20-40 \%$. These uncertainties can only be overcome by including a consistent model for the velocity gradients throughout the $\mathrm{H}_{2} \mathrm{O}$ maser source when deriving the magnetic field strengths derived from circular polarization observations.

When there is a magnetic field gradients along the maser amplification path, $B^{\prime}$ determined from polarization observations of an unsaturated maser (or, more formally, when $R \lesssim \Gamma_{v}$ ), is typically equal to the average field along the maser. When the maser is saturated, the $B^{\prime}$ is dominated by the average magnetic field strength or the average magnetic field angle in the unsaturated maser core. The fractional linear polarization on the other hand, is not affected by velocity or magnetic field gradients. Only a gradient in the angle between the magnetic field and the maser propagation axis alters the linear polarization. In contrast to the circular polarization, the fractional linear polarization is mainly determined by the magnetic field angle in the part of the unsaturated maser where the largest amplification occurs and not by the angle averaged over the entire unsaturated maser region.

Acknowledgements. The author acknowledges the helpfull comments by the anonymous referee. This work has been supported by an EC Marie Curie Fellowship under contract number MEIF-CT-2005010393.

\section{References}

Anderson, N., \& Watson, W. D. 1993, ApJ, 407, 620

Elitzur, M. 1990, ApJ, 350, L17

Fiebig, D., \& Guesten, R. 1989, A\&A, 214, 333

Goldreich, P., \& Keeley, D. A. 1972, ApJ, 174, 517

Goldreich, P., Keeley, D. A., \& Kwan, J. Y. 1973, ApJ, 179, 111

Imai, H., Sasao, T., Kameya, O., et al. 1997, A\&A, 317, L67

Kukolich, S. G. 1969, J. Chem. Phys., 50, 3751

Modjaz, M., Moran, J. M., Kondratko, P. T., \& Greenhill, L. J. 2005, ApJ, 626, 104

Nedoluha, G. E., \& Watson, W. D. 1988, ApJ, 335, L19

Nedoluha, G. E., \& Watson, W. D. 1991, ApJ, 367, L63

Nedoluha, G. E., \& Watson, W. D. 1992, ApJ, 384, 185 (NW92)

Reid, M. J., \& Moran, J. M. 1981, ARA\&A, 19, 231

Richards, A. M. S., Yates, J. A., \& Cohen, R. J. 1999, MNRAS, 306, 954

Sarma, A. P., Troland, T. H., \& Romney, J. D. 2001, ApJ, 554, L217

Sarma, A. P., Troland, T. H., Crutcher, R. M., \& Roberts, D. A. 2002, ApJ, 580, 928

Vlemmings, W. H. T., Diamond, P. J., \& van Langevelde, H. J. 2002, A\&A, 394, 589 (V02)

Vlemmings, W. H. T., van Langevelde, H. J., \& Diamond, P. J. 2005, A\&A, 434, 1029 (V05a)

Vlemmings, W. H. T., \& van Langevelde, H. J. 2005, A\&A, 434, 1021 (V05b)

Watson, W. D., \& Wyld, H. W. 2001, ApJ, 558, L55 